\title{
AVALIAÇÃO DOS PARÂMETROS FÍSICO-QUÍMICAS DO PALMITO DE PUPUNHA (Bactris gasipaes) EM CONSERVA DE $600 \mathrm{ML}$
}

\author{
Georgeano Dantas Maciel ${ }^{1}$, Fernando Berton Zanchi ${ }^{2}$, Raimundo Kennedy Vieira ${ }^{3}$
}

\section{RESUMO}

O presente trabalho tem como objetivo avaliar os parâmetros físico-químicas de palmitos do tipo pupunha picados de três amostras diferentes em conserva de $600 \mathrm{ml}$ comercializadas no município de porto velho do estado de Rondônia verificando seu peso bruto ( $\mathrm{Kg}$ ), espaço livre (m), peso líquido $(\mathrm{Kg})$, peso drenado $(\mathrm{Kg}), \mathrm{pH}$ (potencial hidrogeniônico), e vácuo $(\mathrm{mm} \mathrm{Hg})$ e comparando esses parâmetros com a legislação RDC N 17, de 19 de novembro de 1999. Foram selecionadas três amostras com três lotes diferentes de palmito de pupunha picado sendo denominados marca A, marca B e marca C. e com relação aos parâmetros físico-químicas nenhuma amostra mostrou-se inadequado com relação à legislação vigente. Com isso foram identificadas algumas variações nos dados estatísticos no coeficiente de variação nas marcas B e C indicando uma possível despadronização na produção. No qual conclui-se a necessidade de um maior controle de qualidade na produção, pois algumas variações foram encontradas nos parâmetros analisados, o que interfere diretamente na aceitabilidade do produto.

Palavras chave: Avaliação, Análises físico químicas, Palmito de pupunha picado.

\section{ABSTRACT}

The objective of this study was to evaluate the physicochemical parameters of peach palm-type palm hearts from three different $600 \mathrm{ml}$ canned samples commercialized in the city of Porto do Rondônia, Brazil, by checking their gross weight $(\mathrm{kg})$, free space $(\mathrm{m})(\mathrm{Kg}), \mathrm{pH}$ (hydrogenation potential), and vacuum ( $\mathrm{mm} \mathrm{Hg}$ ), and comparing these parameters with RDC legislation No. 17 of November 19, 1999. Three samples were selected with three batches different from peach palm heartfish being denominated brand A, brand B and brand C. Regarding the physicalchemical parameters, no sample proved to be inadequate in relation to the current legislation. With this, some variations in the statistical data were identified in the coefficient of variation in the marks $\mathrm{B}$ and $\mathrm{C}$ indicating a possible disadronisation in the production. In which the need

\footnotetext{
${ }^{1}$ Pós-Graduação - Mestrado em Engenharia da Produção pela Universidade Federal do Amazonas - UFAM georgeano.maciel@gmail.com

${ }^{2}$ Faculdade de Rondônia - FARO.

${ }^{3}$ Universidade Federal do Amazonas - UFAM.
} 
for a greater quality control in the production is concluded, because some variations were found in the analyzed parameters, which directly interferes in the acceptability of the product.

Key words: Evaluation, Physical chemical analysis, Palm heart of chopped pupunha.

\section{INTRODUÇÃO}

A segurança está profundamente associada à possível presença de perigos, no momento do consumo do alimento e à entrada dos mesmos na cadeia de produção pode dar-se em qualquer etapa. Posteriormente, é primordial que tenha um controle especial no decorrer de toda a cadeia, visando garantir a segurança do alimento, além de combinar esforços de todas as partes que integram o processo (CRIOLLO, 2016)

Segundo Fuzitani e colaboradores (2016), A exploração de palmito no Brasil está deixando de ser uma atividade extrativista para se tornar agrícola, devido ao plantio de espécies de palmeiras, como a pupunheira. O emprego de espaçamentos em plantio apropriados proporciona uma melhor disposição do sistema radicular e melhor condição de exploração do perfil do solo.

O palmito de juçara é basicamente produzido em sistema extrativista, sendo que atualmente só pode ser comercializado mediante a aprovação de rigoroso plano de manejo, o que acarretou redução drástica de produção (PARANÁ, 2013).

A comercialização de vegetais processados, frutas ou legumes, vêm aumentando no mercado brasileiro visto que os consumidores buscam cada vez mais produtos prontos para o consumo devido à praticidade e conveniência encontrada nesses alimentos (SEBRAE, 2014).

Por volta de 1950, o Brasil começou a importar o palmito em conserva, tendo a França como a primeira a obter esse produto, tornando o Brasil, tempos depois, como maior produtor e exportador. Após a obtenção deste título, a Codex Alimentarius buscou elaborar um padrão para identidade e qualidade para o palmito em conserva, que seria utilizado pelos órgãos fiscalizadores, afim de garantir a qualidade e uniformidade nas avaliações (VIEIRA, 2009).

O Brasil é o maior produtor e consumidor de palmito do mundo. Porém, nos últimos anos, o Equador e a Costa Rica assumiram a liderança na exportação, devido ao cultivo de pupunheiras de forma organizada e produção em grande escala e, consequentemente, oferecer o produto com preços mais baixos, assumindo assim a liderança do mercado internacional (SOUSA e colaboradores, 2009). 
A padronização dos processos tem como destino sintetizar a variabilidade dos processos de trabalho sem que a versatilidade seja prejudicada, ou seja, os produtos devem atender às expectativas dos consumidores de forma frequente com o menor custo possível. Os mecanismos de padronização muitas vezes são indispensáveis para uma empresa se manter competitiva no mercado, já que afeta os custos de produção, os prazos de entrega e a satisfação do cliente. (LIMA; CARVALHO JUNIOR, 2012).

Corroborando com essa ideia Pereira e Bueno da Costa (2014) informam que a melhoria da qualidade dos produtos, a redução de custos operacionais e a inovação tecnológica são aspectos importantes para sustentar as estratégias das organizações na disputa por mercados.

De acordo com Maranhão (2016) um dos alimentos mais antigos a que a documentação histórica brasileira se refere é o palmito: de fato, a Carta de Caminha, de abril de 1500, faz menção a ele, como comida dos primitivos habitantes da Terra de Santa Cruz. Diversas espécies foram exploradas ao longo da História, e até muito recentemente a atividade produtora desse ingrediente era exclusivamente a de extração, dada a frequência de palmeiras portadoras de palmito em nossas florestas.

Segundo Rodrigues e colaboradores (2014) a pupunha (Bactris gasipaes var. gasipaes) é a espécie produtora de palmito com maior área cultivada no Brasil, sendo São Paulo e Bahia os maiores produtores. Entretanto, a produção comercial é baixa, em torno de $600 \mathrm{~g}$ por planta.

De acordo com Soares (2011) o consumo "in natura" do palmito deve ser incentivado e estudos estão sendo realizados para aumentar a duração pós-colheita. No entanto, como todo produto vegetal, é perecível e deve ser processado ou consumido em prazo máximo de 4 a 7 dias após a colheita, maiores prazos de prateleira dependem de um processo meticuloso de sanitização e conservação.

A comercialização de vegetais processados, frutas ou legumes, vêm aumentando no mercado brasileiro visto que os consumidores buscam cada vez mais produtos prontos para o consumo devido à praticidade e conveniência encontrada nesses alimentos (SEBRAE, 2014).

De acordo com a Resolução RDC no 18 (1999) a investigação sanitária, a partir dos casos ocorridos de Botulismo, aponta falhas no processo de produção das conservas de palmito, encontrando um grande número de produtos de origem clandestina e sem critérios de controle de segurança e qualidade do produto.

Assim, o Botulismo por ingestão de toxinas presentes em alimentos contaminados produzidos ou conservados de maneira inadequada são de maior predominância em: conservas vegetais, 
principalmente as artesanais (palmito, pequi, picles); entre outros alimentos enlatados e industrializados. (BRASIL, 2006).

Com isso verificando os parâmetros de qualidade é de extrema importância para a investigação de alterações realizadas no decorrer do armazenamento e/ou processamento do palmito em conserva e auxilia também na regulamentação do produto final.

Considerando a importância do controle de qualidade do processamento do palmito, o presente estudo teve como objetivo avaliar os parâmetros físico-químicas de palmitos do tipo pupunha picados de três amostras diferentes em conserva de $600 \mathrm{ml}$ verificando seu peso bruto $(\mathrm{Kg})$, espaço livre (m), peso líquido $(\mathrm{Kg})$, peso drenado $(\mathrm{Kg}), \mathrm{pH}$ (potencial hidrogeniônico), e vácuo $(\mathrm{mm} \mathrm{Hg})$ a uma dada temperatura $\left({ }^{\circ} \mathrm{C}\right)$ e comparando esses parâmetros com a legislação RDC $\mathrm{N}^{\mathrm{o}} 17$, de 19 de novembro de 1999.

\section{MATERIAL E MÉTODOS}

Para o desenvolvimento desta avaliação foram adquiridas 03 amostras/marcas de palmito do tipo pupunha (Bactris gasipaes) picado $600 \mathrm{ml}$. Os procedimentos foram realizados em triplicata com três lotes diferentes para cada marca de palmito de pupunha picado em conserva acidificado com ácido cítrico. Denominados da seguinte forma: marca A, marca B, marca C e seus respectivos lotes: lote 1 , lote 2 e lote 3 respectivamente.

$\mathrm{O}$ pH (potencial hidrogeniônico) foi determinado com auxílio de phmetro (modelo pHBANC II) utilizando-se $10 \mathrm{~g}$ da amostra de palmito triturado em liquidificador juntamente com $20 \mathrm{ml}$ da salmoura (INSTITUTO ADOLFO LUTZ, 1985).

O peso das amostras analisadas foi determinado com auxílio de balança digital de bancada (marca Toledo) e os resultados foram expressos em gramas (g). Foram determinados: peso bruto (frasco, tampa, palmito e salmoura), peso líquido (palmito e salmoura), peso drenado (palmito) e peso da salmoura. Os resultados foram expressos em gramas (g) (BRASIL, 1999a).

O vácuo presente nos frascos das amostras de palmitos analisados foi determinado utilizando um vacuômetro analógico (marca Salvi, com amplitude de -760 a 0 mmHg -30 a 0 pol.Hg). Inicialmente a tampa do frasco foi umedecida ligeiramente com água, o vacuômetro foi comprimido firmemente na tampa, próximo à borda e esta foi perfurada. Dessa maneira, a leitura da deflexão da agulha foi realizada e os resultados foram expressos em milímetro de mercúrio (mmHg). 
O espaço livre, caracterizado pelo espaço entre o palmito juntamente com a salmoura e a tampa do frasco, foi determinado com régua metálica graduada em milímetros ( $\mathrm{mm})$.

\section{RESULTADOS E DISCUSSÃO}

A tabela 1 mostra as análises estatísticas dos parâmetros físico-químicas das amostras A, B e C respectivamente de palmito do tipo pupunha analisadas. Tais parâmetros físico-químicas da tabela são essenciais para atestar a qualidade do palmito sendo, inclusive, exigidas pela legislação RDC No 17, de 19 de novembro de 1999 (BRASIL, 1999a).

Tabela 1 - Análises estatísticas dos parâmetros físico-químicas das amostras A, B e C respectivamente de palmito do tipo pupunha analisadas

\begin{tabular}{|c|c|c|c|c|c|c|c|c|c|c|c|c|}
\hline \multirow{2}{*}{ Variáveis } & \multicolumn{4}{|c|}{ Marca A } & \multicolumn{4}{|c|}{ Marca B } & \multicolumn{4}{|c|}{ Marca C } \\
\hline & Média & $\mathbf{S}^{2}$ & $\mathbf{S}$ & C.V & Média & $\mathbf{S}^{2}$ & $\mathbf{S}$ & C.V & Média & $\mathbf{S}^{2}$ & $\mathbf{S}$ & C.V \\
\hline $\begin{array}{l}\text { Espaço Livre } \\
(\mathbf{m m})\end{array}$ & 19,33 & 0,89 & 0,94 & 4,88 & 16,00 & 48,67 & 6,97 & 43,60 & 12,00 & 4,67 & 2,16 & 18,00 \\
\hline $\begin{array}{c}\text { Peso Bruto } \\
\text { (g) }\end{array}$ & 836,33 & 5,55 & 2,36 & 0,28 & 819,00 & $2.128,67$ & 46,14 & 5,63 & 797,33 & $2.430,89$ & 49,30 & 6,18 \\
\hline $\begin{array}{l}\text { Peso Líquido } \\
\text { (g) }\end{array}$ & 552,33 & 216,22 & 14,70 & 2,66 & 573,67 & 68,22 & 8,26 & 1,44 & 555,33 & $1.448,22$ & 38,06 & 6,85 \\
\hline $\begin{array}{c}\text { Peso Drenado } \\
\text { (g) }\end{array}$ & 263,00 & 160,67 & 12,68 & 4,82 & 222,00 & $1.158,00$ & 34,03 & 15,32 & 233,00 & 326 & 18,05 & 7,75 \\
\hline pH & 3,73 & 0,0022 & 0,0473 & 1,26 & 3,70 & 0,02 & 0,14 & 3,82 & 3,63 & 0,03 & 0,17 & 4,68 \\
\hline $\begin{array}{c}\text { Vácuo } \\
\text { (mmHg) }\end{array}$ & 430 & & & & 450 & & & & 480 & & & \\
\hline
\end{tabular}

S 2.: variância; S.: desvio padrão; C.V.: coeficiente de variação (\%)

De acordo com a RDC n ${ }^{\circ} 17$ de 1999 o vidro de vidro deve ser cheio com palmito e líquido de cobertura e ambos devem ocupar no mínimo 90\% da capacidade de água do recipiente. Dessa maneira, nota-se que dentre todas as médias das amostras analisadas apresentaram conforme à legislação acima.

A média dos $\mathrm{pH}$ das amostras dos palmitos analisados variou de 3,63 a 3,73 comprovando que o processo de acidificação possui alterações entre as diferentes indústrias. Conforme A Legislação RDC No 17, de 19 de novembro de 1999 específica para este tipo de alimento produzido o pH deve estar a 4,5 ou valor menor. Além disso, as indústrias devem possuir como meta o pH no valor de 4,3, para que o equilíbrio entre solução e palmito não ultrapasse o valor 
de 4,5 considerado como limite crítico. Dessa maneira, nota-se que dentre todas as amostras analisadas apresentaram conforme à legislação citada anteriormente.

As embalagens de palmito devem ser fechadas hermeticamente evitando que contaminações físicas, químicas e microbiológicas ocorram no produto e este possa ser oferecido com segurança aos consumidores (BRASIL, 2016). Dessa maneira, o vácuo das embalagens deve ser avaliado como um parâmetro da garantia de qualidade.

A média dos vácuos avaliados nas embalagens de vidro de $600 \mathrm{ml}$ dos palmitos de pupunha apresentaram variação de 430 a 480 mmHg. De acordo com a Resolução no 85 de 2016 os frascos de vidro devem conter, no mínimo, vácuo de $180 \mathrm{mmHg}$, demonstrando que todas as amostras se apresentaram adequadas à legislação vigente (BRASIL, 2016).

Analisou-se que a média do peso do palmito (peso drenado) variou de $222 \mathrm{~g}$ a $263 \mathrm{~g}$ e a média da salmoura variou de $289,33 \mathrm{~g}$ a $351,67 \mathrm{~g}$, mostrando que existe variabilidade no envase entre diferentes empresas. Estudo de Raupp et al. (2004) observou que houve uma média 294,4 g de palmito e 247,9 g de salmoura, valores estes relacionados a média do peso drenado maior e menores relacionados a média da salmoura quando comparados ao estudo em questão.

De acordo com a RDC n 18 de 1999 os vidros de palmito de $600 \mathrm{ml}$ devem conter de 200g a $300 \mathrm{~g}$ de palmito drenado, demonstrando assim, que todas as amostras analisadas estavam adequadas (BRASIL, 1999b).

\section{CONCLUSÃO}

O controle de qualidade do palmito em conserva é uma atividade complexa, conforme leis federais, estaduais e municipais, bem rigorosa com o intuito de garantir produtos alimentícios que não sejam prejudiciais à saúde, devendo estar em boa qualidade ao consumidor e em quantidade adequada as especificadas pelos fabricantes.

Com relação aos parâmetros físico-químicas nenhuma amostra mostrou-se inadequado com relação à legislação vigente. Porém mesmo as amostras contendo de $200 \mathrm{~g}$ a $300 \mathrm{~g}$ de palmito drenado fora observadas diferenças significativas no coeficiente de variação nas médias das marcas B e C indicando uma possível despadronização nas mesmas.

Conclui-se com este estudo que as indústrias de palmito do tipo pupunha picado comercializado na cidade de Porto Velho necessitam de um maior controle de qualidade na produção, pois algumas variações foram encontradas nos parâmetros analisados, o que interfere diretamente na aceitabilidade do produto. 


\section{REFERÊNCIAS}

BRASIL. Ministério da Saúde. Agência Nacional de Vigilância Sanitária - ANVISA.

Resolução $n^{\circ}$ 85, de 27 de junho de 2016. Altera a Resolução da Diretoria Colegiada - RDC $n^{\circ} 17$, de 19 de novembro de 1999, que dispõe sobre o padrão de identidade e qualidade para palmito em conserva. Disponível em <www.lex.com.br/legis_27161292_RESOLUCAO_N_85_DE_27_DE_JUNHO_DE_2016.as px>. Acesso em 10 mai. 2019.

BRASIL. Agência Nacional de Vigilância Sanitário. RDC ${ }^{\circ} 17$ de 19 de novembro de 1999. Regulamento Técnico referente ao Padrão de Identidade e Qualidade para Palmito em Conserva. Disponível em: 〈http://portal.anvisa.gov.br/wps/wcm/connect.pdf $>$. Acesso em: 16 de mai. 2014.

BRASIL. Resolução RDC n ${ }^{\circ}$, de 19 de novembro de 1999. D.O.U. - Diário Oficial da União;1999. Disponível em:<

portal.anvisa.gov.br/wps/wcm/connect/.../RDC_18.pdf?MOD=AJPERES> Acesso em 16 de mai.2019.

BRASIL. Resolução RDC no 81, de 14 de abril de 2003. D.O.U. - Diário Oficial da União; 2003. Disponível em:

http://portal.anvisa.gov.br/wps/wcm/connect/b151928047458937932dd73fbc4c6735/RDC_81 .pdf?MOD=AJPERES Acesso em 15 de mai.2019.

CRIOLO, Luisa Fernanda Rivera. Monitoramento e avaliação da regulamentação sobre rotulagem de alimentos alergênicos no Brasil: proposição de indicadores e métricas. Dissertação (mestrado) - Pontifícia Universidade Católica do Rio de Janeiro, Programa de Pós-Graduação em Metrologia, 2016.

EMBRAPA. Manejo de Pupunheira (Bactris gasipaes Kunth) para Palmito em Áreas de Restrições Hídricas. Colombo, dez. 2004. Disponível em: < http://ainfo.cnptia.embrapa.br/digital/bitstream/CNPF-2009-09/38125/1/circ-tec89.pdf > Acesso em 21 de mai. 2019.

FUZITANI, E. J.; SANTOS, A. F. dos; NEVES, E. J. M.; DAMATTO JÚNIOR, E. R.; NOMURA, E. S.; 2014. Desenvolvimento fenológico de pupunheiras para produção de palmito em diferentes espaçamentos. Horticultura Brasileira 31: S1220 - S1226.

IAL - Instituto Adolfo Lutz. Normas analíticas do Instituto Adolfo Lutz: métodos químicos e físicos para análise de alimentos. São Paulo. 4 ed. v. 1. p.23, 2005.

KALIL FILHO, A. N.; GUIOTOKU, M. Influência do tempo na perda de umidade em palmito pupunha (Bactris gasipaes Kunth. var. gasipaes Henderson) armazenados em geladeira. ISSN 1980-3982 Colombo, PR Dezembro, 2014.

LIMA, B. T. P; CARVALHO JÚNIOR, L. C. A importância da padronização de processos e gerenciamento no setor de fast-food em Florianópolis. VI EEC.

Joinville, abr. 2012. Disponível em: Acesso em: 27 abr. 2019. 
MARANHÃO, Ricardo. O Palmito Pupunha, a Gastronomia e o Meio Ambiente. Revista.pdf. Revista Rosa dos Ventos, 4(III), p.352-368, jul-set, 2012.

PEREIRA, C. R., \& Bueno da Costa, M. A. (2014). Um modelo de simulação de sistemas aplicado à programação da produção de um frigorífico de peixe.

Revista Produção Online, 12(4), 972. https://doi.org/10.14488/1676-1901.v12i4.994.

SOARES, Naisy. Silva.; SOUSA, Eliane. Pinheiro de.; CORDEIRO, Sidney. Araujo.; SILVA, Márcio. Lopes. Competitividade do palmito de pupunha no Brasil em diferentes sistemas de produção. Arvore, Viçosa, ano 2011, V.35, n6, Nov/dez 2011.

VIEIRA, Priscila da Silva. Avaliação do Palmito Pupunha (Bactris gasipaes Kunth) Processado por Radição Ionizante. Dissertação. São Paulo, 2009. Disponível em:< http://www.teses.usp.br/teses/disponiveis/85/85131/tde-17112009-154316/pt-br.php> Acesso em 28 de março de 2019.

VIEIRA, Ruth Lemos Vanconcelos. Efeito da Acidificação com Diferentes ácidos sobre as Características sensoriais e inibição de Clostridium Botulinum no Palmito de Pupunha em Conserva. Dissertação. Recife, 2004, pg 11.

FUZITANI, E. J.; SANTOS, A. F. dos; NEVES, E. J. M.; DAMATTO JÚNIOR, E. R.; NOMURA, E. S.; 2014. Desenvolvimento fenológico de pupunheiras para produção de palmito em diferentes espaçamentos. Horticultura Brasileira 31: S1220 - S1226.

RODRIGUES, H. S., Cruz, C. D., Macêdo, J. L. V. de, Resende, M. D. V. de, Lopes, R., \& Borém, A. (2017). \&lt;b\&gt;Genetic variability and progeny selection of peach palm via mixed models (REML / BLUP). Acta Scientiarum. Agronomy, 39(2), 165. https://do

SOARES, Naisy. Silva.; SOUSA, Eliane. Pinheiro de.; CORDEIRO, Sidney. Araujo.; SILVA, Márcio. Lopes. Competitividade do palmito de pupunha no Brasil em diferentes sistemas de produção. Arvore, Viçosa, ano 2011, V.35, n6, Nov/dez 2011. 